\title{
OXYGEN TENSION OF TISSUES BY THE POLAROGRAPHIC METHOD. V. THE RATE OF MOVEMENT OF OXYGEN FROM THE PERIPHERAL ARTERY TO THE SKIN ${ }^{1}$
}

\author{
BY RAYMOND PENNEYS AND HUGH MONTGOMERY \\ (From the Peripheral Vascular Section, Robinette Foundation, Medical Clinic, Hospital of the \\ University of Pennsylvania, Philadelphia, Pa.)
}

(Submitted for publication August 1, 1952; accepted September 12, 1952)

By means of the oxygen electrode (1), which measures polarographically the oxygen tension of living tissues in situ, several workers have reported a time lag in the changes of tissue oxygen after arterial blood oxygen changes. Behrmann, Griest, Mangun, and Hartman showed in hypoxemia studies in dogs that the changes in oxygen tension of animal viscera lagged measurably behind the changes of oxygen in the peripheral arterial blood (2). Experiments have been made with pressure cuffs in human extremities. On deflating a cuff the oxygen tension of the skin of the extremity recovered some time after the skin became fully pink (3). In another set of human experiments, changes in skin oxygen tension lagged considerably behind the changes in blood arterial oxygen saturation (4).

In none of these studies was the lag between the initial changes of arterial and tissue oxygen compared. It is the purpose of this report to describe this time lag by comparing the exact time of first appearance of oxygen in skin tissue (polarographic measurement) with the exact time of first appearance of oxygen in the peripheral arterial blood (oximetric measurement) and to correlate this "saturation-tension lag" with gross changes in peripheral blood flow. The increment in blood oxygen saturation was furnished by inhalation of oxygen by the subject.

\section{METHODS}

Subjects were either white healthy adults or white patients with peripheral arterial disease. A Millikan oximeter was attached to the ear and "arterialization" of the blood in the ear was accomplished by heat, in the usual manner (5). Oxygen electrodes were inserted in the skin of one or more extremities (3). Estimates of skin blood flow were made by taking the temperature of the skin adjacent to the electrodes and of air in the room, all

1 This work was supported by the Life Insurance Medical Research Fund. obtained by means of thermocouples. Room temperature was constant throughout each experiment. Some of the experiments were conducted in a thermoregulated "cool room." Oxygen-administering apparatus consisted of a demand valve attached to a mouth-piece through a threeway stop-cock.

After the readings of skin temperature, of oxygen electrode and of oximeter became stable, the nose of the subject was obstructed by a clip, the valve and attached tubing were flushed out with oxygen, oxygen was administered by quickly turning the stop-cock just prior to the beginning of a normal inspiration and "zero" time was set with the first "hiss" of the demand valve. The very first changes of the readings by oximeter and by oxygen electrode were obtained by noting the first increment in current shown by each galvanometer. Subsequent changes were noted at approximately five-second intervals for a period of at least one minute. In order to make frequent oxygen tension readings the electrode switch (3) was kept continually closed, starting one minute prior to the beginning of oxygen inhalation.

\section{RESULTS}

\section{A. Warm skin, with normal blood flow}

For convenience, measurements of the oxygen tension were usually made in the forearm. When made in the hand, foot or digits, the tension lag did not differ significantly unless blood flow was different.

Most of the subjects were normal individuals. A few were patients with peripheral arterial disease who had approximately normal peripheral arterial blood flow as measured by skin temperature. The average temperature of the skin of extremities of all the subjects was $31.7^{\circ} \mathrm{C}$., and of the room $24.6^{\circ} \mathrm{C}$. Heat was added to the body, if needed, to maintain the skin temperature of the extremity at the desired level.

In these experiments the first change of the arterial oxygen saturation in the ear, occurred, as expected (6), in an average of 8.6 seconds after the start of oxygen inhalation. The first change in the oxygen tension (polarographic method) of 
OXYGEN TENSION OF TISSUES. V

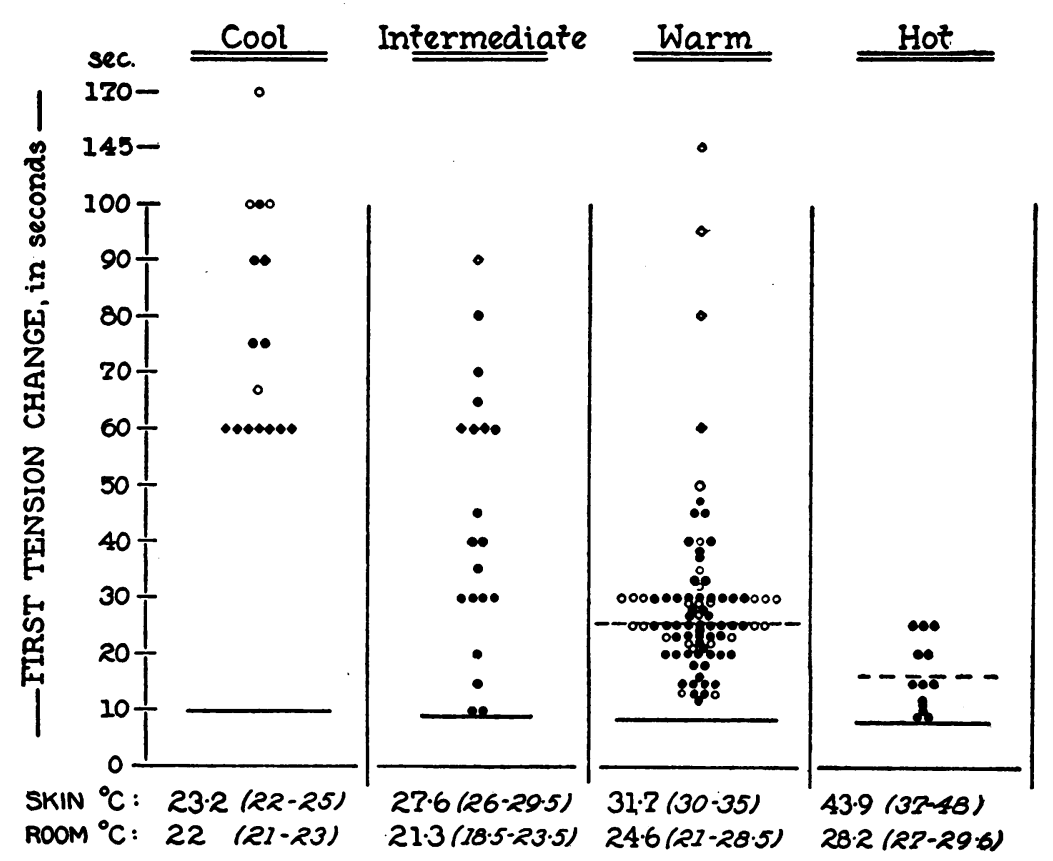

Fig. 1. The First Change in Oxygen Tension of Cool, Intermediate, Warm and Hot Skin; All Experrments

Empty symbols indicate observations before November 17, 1950; solid symbols, after that date (see text).

$\diamond, \diamond$ indicate that no oxygen tension change had taken place by this time and that the experiment was carried no further. Such values were not used to derive mean values.

- - - is mean value of first oxygen tension change, as measured in the skin of an extremity by polarographic method. oximeter.

is mean value of first oxygen saturation change, as measured by the

Temperature of HOT skin is that of the water when direct heating was obtained by immersion in water bath (see Figure 5).

the warm skin of the forearm, however, took place in an average of 25.5 seconds, lagging behind the first change of the arterial blood by 16.9 seconds (Figures 1, 2).

Special mention should be made of the early group of experiments on normal subjects (done before November 17, 1950 in which an occasional, considerably longer delay of the first change in tension was noted. In three of these 28 experiments, the first change in the oxygen tension of the skin was very slow and indefinite, not occurring within 60 seconds. Possibly the way of inserting the electrodes was responsible for the results in these three instances. These 28 early experiments are shown in Figure 1. In all subsequent 57 experiments (after November 17, 1950), when special attention was given to the insertion of the elec- trodes in the skin, only one such unusually long delay was encountered (Figure 1).

\section{B. Cool skin, with reduced blood flow}

To investigate the role of peripheral blood flow on the time interval between change in oxygen saturation of arterial blood and in oxygen tension of skin, measurements were made in the skin of extremities of six patients with peripheral arterial disease, and of one normal subject, before and after reflex vasodilatation caused by heating the body. Before vasodilatation, the first change of the arterial saturation following oxygen inhalation took place in an average of 9.8 seconds, but the first change of the oxygen tension of the cool skin was considerably delayed. When the skin temperature was $23.2^{\circ} \mathrm{C}$., and the room temperature 


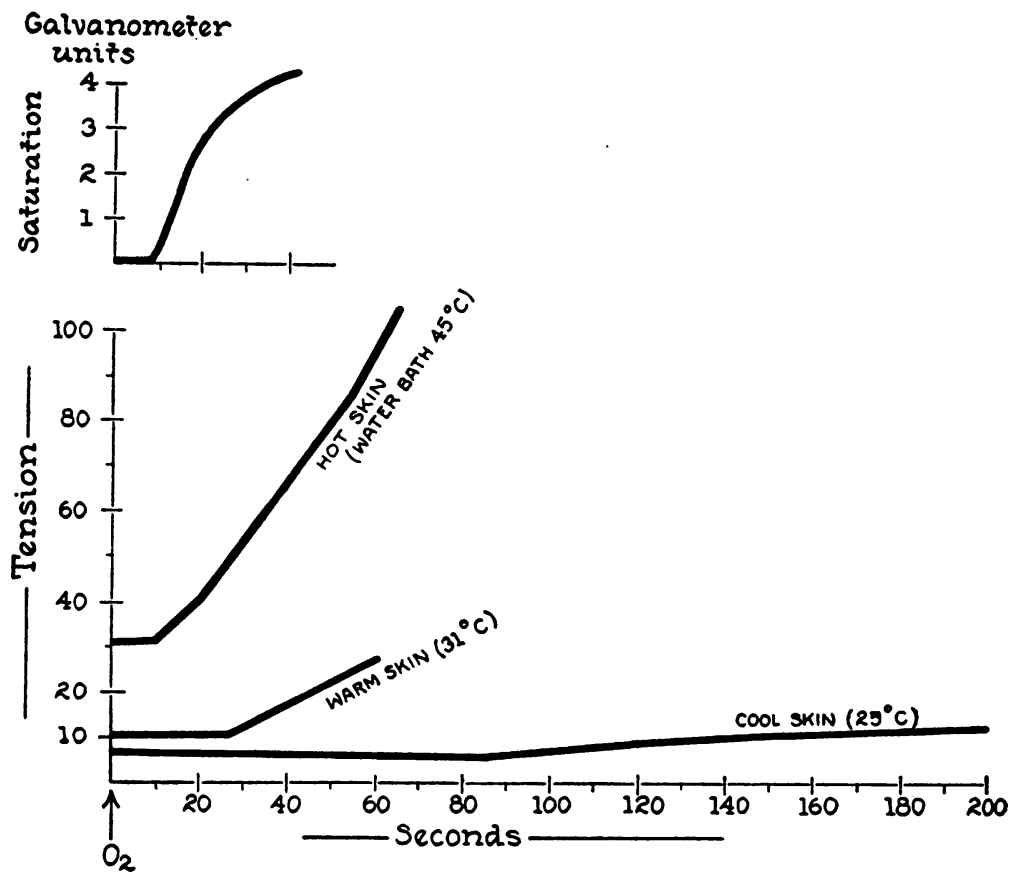

Fig. 2. The First Change in the Arterial Oxygen Saturation of the Blood and in the Oxygen Tension of Warm, Cool, and Hor Skin. Three Typical Experiments

Saturation changes are essentially the same in all experiments. " $\mathrm{O}_{2}$ " indicates beginning of inhalation of oxygen. The experiments are on three different subjects.

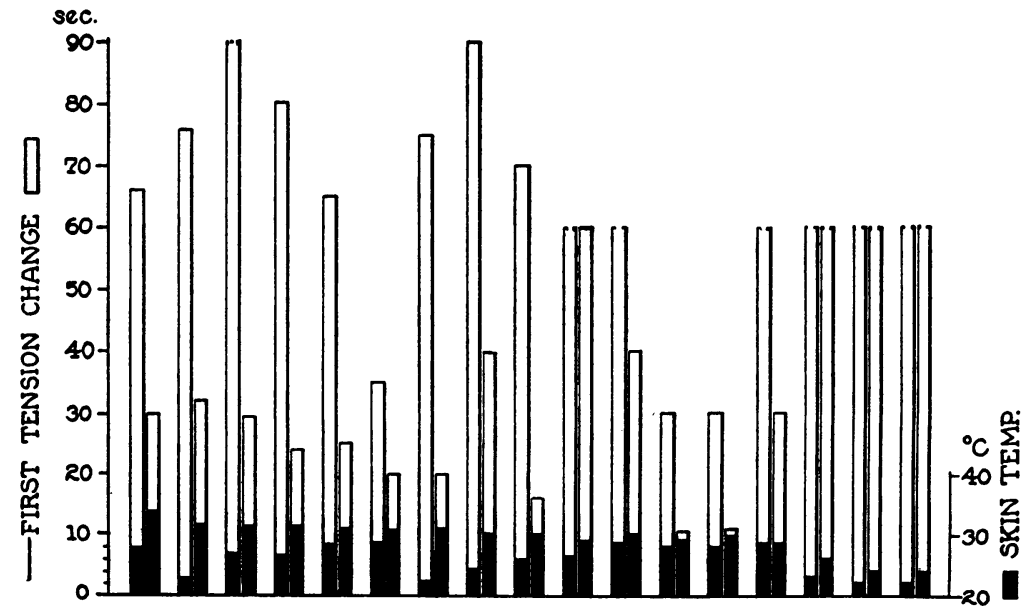

Fig. 3. The First Change in Oxygen Tension of the Skin Before and After Reflex Vasodilatation

Each pair of bars represents values obtained with one electrode, remaining undisturbed throughout the experiment. The first bar of each pair represents the value obtained before reflex vasodilatation was attempted by body warming; the second bar, the value after body warming. Broken line at top of bar means experiment was terminated at this time without a skin oxygen tension change having as yet been detected. 
$22^{\circ} \mathrm{C}$., a first tension change failed to occur within 60 seconds. In those instances, after body warming, in which the previously cool skin of an extremity became warm by reason of reflex vasodilatation an average time of 26 seconds was necessary for the first change of the oxygen tension. In those patients in whom a significant degree of reflex vasodilatation failed to occur, and the skin remained cool, the time necessary for the first change of the oxygen tension remained over 60 seconds; with incomplete vasodilatation (skin temperature from 26 to $29.5^{\circ} \mathrm{C}$.), the first tension change was variable (Figures 1,2,3).

Studies before and after body warming of a patient with symptoms of ischemia in the left leg as a result of occlusion of the popliteal artery are shown in Figure 4. The other extremities were unaffected. As blood flow changed, or remained unchanged as in the case of the left leg, corresponding changes in the time lag resulted.
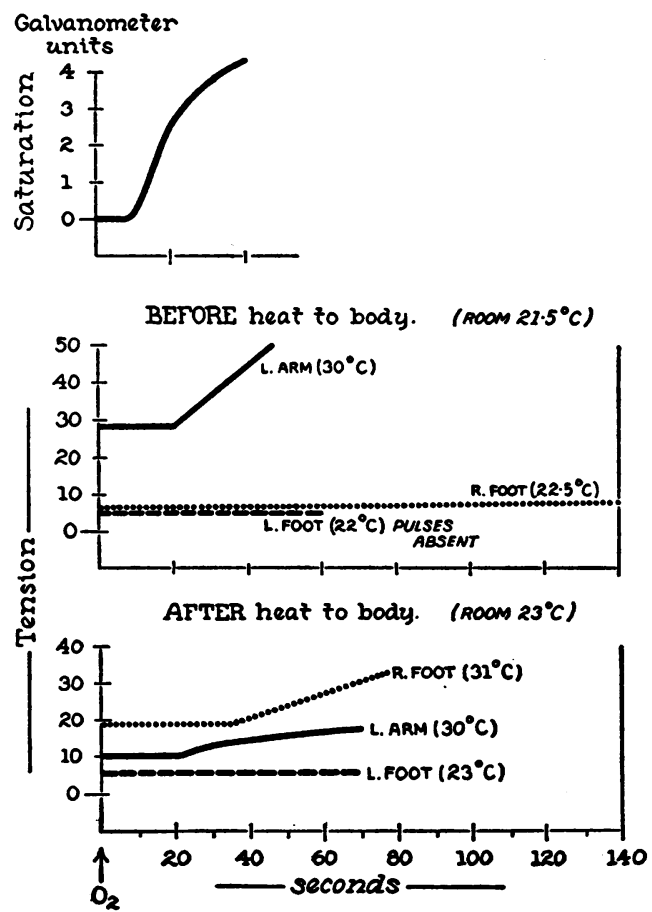

Fig. 4. The First Change of the Blood Arterial Saturation and of the Oxygen Tension of the Skin, Before and After Reflex Vasodilatation, by Heat to Body, in a Patient with Peripheral Arterial Disease of the Left Leg (Occlusion of Left Popliteal. ARTERY)

Saturation changes, shown in the upper graph, are essentially the same before and after reflex vasodilatation.

\section{Hot skin, with extremely rapid blood flow}

To investigate the role of extremely rapid peripheral blood flow on the saturation-tension lag, the hands of several normal subjects, having reflex vasodilatation, were further heated by directly applying hot air or hot water. As the heat was increased the lag was reduced (Figures 1, 2, 5). In five hot air experiments, air temperature was maintained at a minimum of $50^{\circ}$ C., raising the skin temperature from 35 to $44^{\circ} \mathrm{C}$. (average). The first change in the dygen tension of the skin took place in an average of 21 seconds. In the three hot water experiments, water with average temperature of $43.9^{\circ} \mathrm{C}$. was used. The first change in the oxygen tension of the hot skin took place in an average of 12 seconds. In two of the latter experiments in which the water temperature was $47^{\circ} \mathrm{C}$., oxygen appeared in the skin in 9 and 11 seconds, almost simultaneously with its detection by the oximeter, on the ear.

In no case was the time of the first oxygen saturation change altered significantly from that in previous experiments, averaging 8 seconds.

\section{Further investigation of the saturation-tension lag}

Several small groups of experiments were performed to investigate further the factors responsible for the time lag in the appearance of oxygen in the skin after its appearance in the peripheral arterial blood:

1. Time required for oxygen to travel from the capillaries to the electrode tip. In the preceding experiments the saturation-tension lag indicates the time required for oxygen to travel through the distal portion of the peripheral artery, arterioles, capillaries and extravascular tissue to the tip of the oxygen electrode. A few experiments were, therefore, carried out to determine what portion of this time was required for the movement of oxygen from the capillaries to the electrode tip. When a blood pressure cuff is inflated on an arm to above systolic pressure for approximately 10 minutes, the skin loses its pink color and the oxygen in the hand is much depleted. When the cuff is released the skin becomes pink as a result of oxygenated blood entering the cutaneous capillaries. The oxygen tension rises shortly thereafter to its original value (3). 


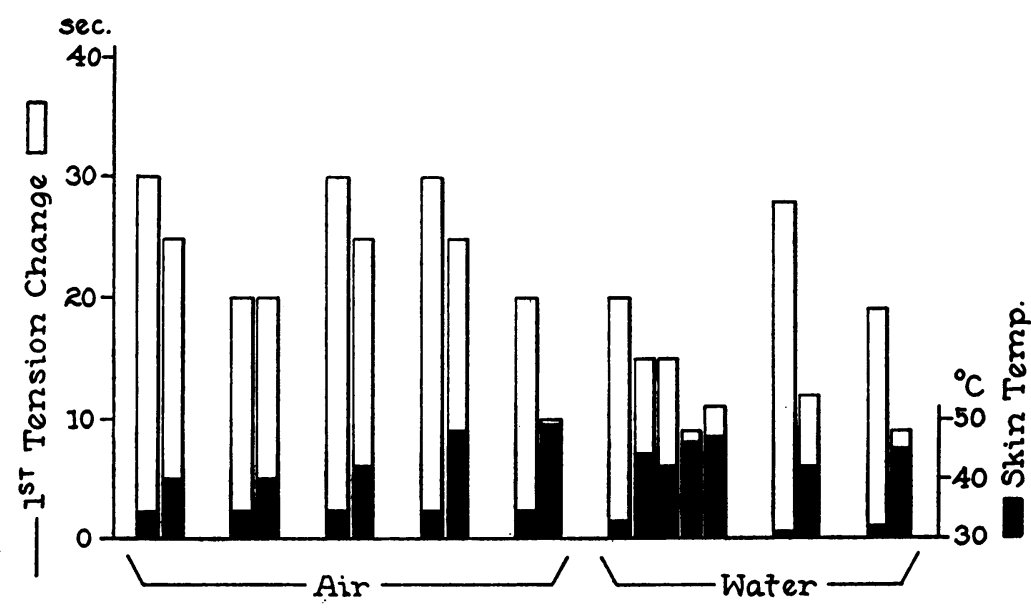

Fig. 5. The First Change in Oxygen Tension of the Skin Before and After Direct Heating of the Skin

Eight experiments are represented. Each group of bars represents the values of one experiment, with the electrode remaining undisturbed throughout the experiment. The first bar of each group shows the value in the warm, vasodilated skin, before direct heating. The remaining bar (or bars) are the values obtained after direct heating of the skin. "Air," heated by hot air; "Water," by hot water.

With this in mind, three electrodes were placed in the skin of fingers. A blood pressure cuff on the upper arm was inflated for ten minutes and suddenly released. In three experiments on the same subject, pink color was clearly apparent in 2 to 2.5 seconds, whereas the first change in tension required 10,12 , and 25 seconds, respectively, in the three electrodes. Later in these same experiments, with electrodes undisturbed, and with no arterial compression, oxygen was inhaled (usual experiment) and the increase in oxygen tension of the skin occurred in 15,15 , and 30 seconds, respectively.

In two other experiments, oxygen was inhaled for several minutes while a cuff was inflated, and the cuff was then released. The inhalation of oxygen did not change the lag; the pink color appeared in 4 seconds, whereas the first increase in tension required 27 and 34 seconds after release of the cuff.

2. Effect of changing temperature of skin in a leg with ischemia. An experiment was conducted in order to study the effect of changes in skin temperature, per se, on the saturation-tension lag. A severely ischemic foot, whose greatly reduced capacity for blood flow had been demonstrated by a reflex vasodilatation test (skin temperature equal to room temperature) and by posterial tibial nerve block, was tested at room temperature $\left(27^{\circ} \mathrm{C}\right.$. $)$, and after immersion in water at $37^{\circ} \mathrm{C}$. At both temperatures the lag remained greater than 60 seconds.

3. Inhalation of a low oxygen gas mixture. In three experiments, the first changes of the oxygen tension of the warm normal skin after the inhalation of 10 per cent $\mathrm{O}_{2}$ were compared with those after the inhalation of pure $\mathrm{O}_{2}$. The time of first change after the low oxygen inhalation $(15,25$, and 45 seconds) was approximately the same as that after inhalation of pure $\mathrm{O}_{2}(20,23$, and 45 seconds).

\section{E. Incidental observations (unrelated to the satu- ration-tension lag)}

1. The degree of increase of oxygen tension of cool, vasoconstricted skin with continued oxygen inhalation. Since the oxygen tension of such skin fails to increase within 60 seconds of oxygen inhalation (Figure 1), two extended experiments were conducted on cool skin. One lasted for six minutes, with skin temperature $22.5^{\circ} \mathrm{C}$., room temperature $22^{\circ} \mathrm{C}$. The oxygen appeared in the skin in 75 seconds and the percentage increase at the end of two and six minutes was 16 per cent 
and 33 per cent, respectively. At the end of an experiment lasting ten minutes, on another subject with skin temperature $22^{\circ} \mathrm{C}$., and room temperature $23^{\circ} \mathrm{C}$., oxygen appeared in the skin in 100 seconds and the percentage increase at the end of two and one-half minutes and ten minutes was 6 per cent and 30 per cent, respectively.

2. Increase of oxygen tension of reflexly vasoconstricted and iced skin with continued oxygen inhalation. In one experiment the skin temperature of reflexly vasoconstricted skin of the foot of a normal subject was further lowered to less than $15^{\circ} \mathrm{C}$. by the direct application of ice, producing the bright pink color characterized by Lewis as the red reaction to cold (7). Oxygen inhalation for five minutes failed to cause any change in oxygen tension.

3. Increase of oxygen tension of hot, vasodilated skin with continued oxygen inhalation. In three experiments there was a great increase in oxygen tension of skin under the combined effects of reflex vasodilatation and direct application of heat and oxygen inhalation. In two instances in which the water temperature was $47^{\circ} \mathrm{C}$., the oxygen tension was increased with oxygen inhalation by 75 per cent in 30 and 40 seconds. In a third experiment, water temperature $45^{\circ} \mathrm{C}$., it was increased by 218 per cent in one minute (Figure 2 ).

4. A comparison of oxygen tension of vasoconstricted and vasodilated skin. In six experiments in which cool skin was warmed from an average temperature of $25^{\circ} \mathrm{C}$. to $30.5^{\circ} \mathrm{C}$. by peripheral vasodilation resulting from heating the body (room temperature $22^{\circ} \mathrm{C}$.), the oxygen tension of the skin was increased approximately by 270 per cent of the initial value, as in experiments previously reported (3).

\section{DISCUSSION}

The time of arrival of the first increment of oxygen in the blood of the great arteries of an extremity is probably very nearly the same as the time of arrival at the oximeter on the ear. The great arteries of an extremity are a little more distant from the heart than is the ear, but the increment shown by the oximeter measures the oxygen increment in ear capillaries, which may be a second or so later than that in the carotid artery. The average first increment of arterial oxygen saturation detectable by the oximeter following the first inhalation of oxygen, was about $9 \mathrm{sec}-$ onds throughout all experiments. Our measurements indicate that oxygen entered the skin some 16 seconds after its appearance in the arterial blood of the ear when the skin was comfortably warm, and its circulation correspondingly fast. This delay occurred in such warm skin whether the subjects were normals or were known to have spastic or occlusive peripheral arterial disease if reasonably fast circulations were maintained in the extremity.

This observation shows that there is a significant, and sometimes conspicuous, delay in the movement of oxygen from the great vessels of an extremity to the skin of the extremity. Under these various conditions, therefore, the time required for oxygen to travel with the blood in the large arteries of an extremity, through the arterioles and capillaries and into the skin tissue is about twice the time required for its entrance into alveoli, diffusion into pulmonary capillaries, its travel through pulmonary vein, left heart, carotid artery, and its entrance into the dilated minute vessels of the ear.

Reflex vasoconstriction in an extremity delayed the first appearance of oxygen in the skin of the extremity far beyond 16 seconds. It did not affect the time of first appearance of oxygen in the great vessels. Consequently, the greatest part of the delay in movement of oxygen in the extremities seems to have occurred in the minute vessels, capillary walls, and extravascular tissues. Further evidence that this is the site of delay comes from experiments in which peripheral vasomotor tone was greatly reduced by a combination of reflex vasodilatation and extreme direct heating. With the minute vessels widely dilated, oxygen entered the skin of the extremities some two seconds after its first appearance in the ear.

The few experiments described in "D. 1." of "Results" were attempts to learn degrees of delay in the extravascular tissue in contradistinction to degrees of delay in the minute vessels. When the cuff was released, oxygenated blood was visible in fingers in about two seconds whereas its detection by the electrode was at least six seconds later. This delay of six seconds is explainable only by time taken for diffusion of oxygen from minute vessels to the electrode. This diffusion time was studied only in warm skin under the particular set 
of circumstances, and was not studied further. Doubtless it varies with the number and size of open capillaries, just as the delays of oxygen transport within vessels vary with vasomotor tone.

\section{SUMMARY}

1. The rate of movement of oxygen was studied by administering pure oxygen by inhalation and noting its very first appearance in the peripheral arterial blood and in the skin.

2. Its appearance in the arterial blood was measured by means of the Millikan oximeter attached to the ear, and in the skin of an extremity by means of the oxygen electrode.

3. Blood flow to the skin of the extremities was altered over a wide range in normal subjects and in patients with peripheral arterial disease.

4. The delay in the movement of oxygen from the peripheral artery to the warm vasodilated skin (average 16 seconds) was markedly increased in cool, vasoconstricted skin, and was conspicuously decreased in hot, extremely vasodilated skin. Evidence is cited that the greatest part of the delay in the movement of oxygen occurs in the minute blood vessels, capillary wall and/or extravascular tissue.

5. In a few related experiments, in warm vasodilated skin, it was also shown that (a) a considerable portion of the delay was due to the time required for oxygen to travel from the capillary, through the extravascular tissue, to the tip of the electrode; (b) 10 per cent oxygen inhalation was followed by the same changes as pure oxygen; and (c) the temperature change of the skin, accompanying blood flow change, did not, per se, have any great affect on the rate of movement of oxygen.
6. Finally, some incidental observations on the effect of continued oxygen inhalation on the percentage rise of the oxygen tension of reflexly vasoconstricted, directly chilled, and directly heated skin are reported.

\section{ACKNOWLEDGMENT}

The authors would like to express their appreciation for the assistance rendered by Miss Diane Grabosky and Miss Ann Sayen.

\section{REFERENCES}

1. Davies, P. W., and Brink, F., Jr., Physical instruments for the biologist: Microelectrodes for measuring local oxygen tension in animal tissues. Rev. Scient. Instruments, 1942, 13, 524.

2. Behrmann, V. G., Griest, W. D., Mangun, G. H., and Hartman, F. W., Comparison of arterial $\mathrm{O}_{2}$ saturation with tissue $\mathrm{O}_{2}$ levels. Federation Proc., 1950, 9, 10.

3. Montgomery, H., and Horwitz, O., Oxygen tension of tissues by the polarographic method. I. Introduction: Oxygen tension and blood flow of the skin of human extremities. J. Clin. Invest., 1950, 29, 1120.

4. Penneys, R., Oxygen tension of the tissues by the polarographic method. IV. Skin oxygen tension vs. arterial oxygen saturation; relationship to oxyhemoglobin dissociation curve. J. Clin. Invest., 1952, 31, 204.

5. Millikan, G. A., Physical instruments for the biologist: The oximeter, an instrument for measuring continuously the oxygen saturation of arterial blood in man. Rev. Scient. Instruments, 1942, 13, 434.

6. Lindgren, I., Continuous measurement of arterial oxygen saturation in man. Cardiologia, 1948, 13, 226.

7. Lewis, T., Vascular Disorders of the Limbs described for practitioners and students. London, The Macmillan Co., 1936, page 9. 